\title{
Impedance Spectroscopy Characterization of Oxygen Transport in Low- and High-Pt Loaded PEM Fuel Cells
}

\author{
Tatyana Reshetenko ${ }^{\mathrm{a}, *, \mathrm{z}}$ and Andrei Kulikovsky $\oplus^{\mathbf{b}, \mathbf{c}, *, \mathrm{z}}$ \\ ${ }^{a}$ Hawaii Natural Energy Institute, University of Hawaii, Honolulu, Hawaii 96822, USA \\ ${ }^{b}$ Institute of Energy and Climate Research, Electrochemical Process Engineering, Forschungszentrum Jülich GmbH, \\ D-52425 Jülich, Germany \\ ${ }^{c}$ Research Computing Center, Lomonosov Moscow State University, 119991 Moscow, Russia
}

\begin{abstract}
We report fitting of the physics-based model for the cathode side impedance to the experimental spectra of low-Pt loaded (0.1/0.1 $\left.\mathrm{mg}_{P t} \mathrm{~cm}^{-2}\right)$ and high-Pt loaded $\left(0.4 / 0.4 \mathrm{mg}_{P t} \mathrm{~cm}^{-2}\right)$ PEM fuel cells measured in the range of current densities from 50 to 400 $\mathrm{mA} \mathrm{cm}{ }^{-2}$. Fitting allowed us to separate the oxygen diffusion coefficients in the catalyst layer $D_{o x}$ and in the gas-diffusion layer $D_{b}$, and the respective mass transfer coefficients of the electrodes of both types. In the low-Pt electrode, $D_{o x}$ is an order of magnitude lower, than in the high-Pt electrode; however, due to 4-fold difference in the electrode thickness, the respective mass transfer coefficients are close to each other. In both the electrodes, the oxygen diffusion and the mass transfer coefficients in the GDL are nearly the same and they are much higher, than the respective coefficients in the CCLs. The ORR Tafel slope and $D_{o x}$ exhibit linear growth with the cell current density; both effects could be attributed to "cleaning" of Pt surface from oxides at lower cell potential.

(C) The Author(s) 2017. Published by ECS. This is an open access article distributed under the terms of the Creative Commons Attribution 4.0 License (CC BY, http://creativecommons.org/licenses/by/4.0/), which permits unrestricted reuse of the work in any medium, provided the original work is properly cited. [DOI: 10.1149/2.1131714jes] All rights reserved.

(cc) BY
\end{abstract}

Manuscript submitted October 9, 2017; revised manuscript received November 20, 2017. Published December 12, 2017.

A future of polymer electrolyte membrane fuel cells (PEMFCs) on the automotive market depends on their reliability and cost. At present, the cathode side of a typical PEMFC used in a 50-kW stack employs Pt loading of $0.4 \mathrm{mg}_{P t} \mathrm{~cm}^{-2}$. With the power density on the order of a $0.5 \mathrm{~W} \mathrm{~cm}^{-2}$, this requires $40 \mathrm{~g}$ of expensive Pt per stack. Clearly, lowering of Pt content while keeping the cell performance is a problem of highest priority for the fuel cell industry. US Department of Energy has set a target of $0.125 \mathrm{~g}_{P t} / \mathrm{kW}$ by the year 2020; at present, typical Pt loading is about $1 \mathrm{~g}_{P t} / \mathrm{kW}$.

The hydrogen oxidation on the anode side is fast and it typically requires much less $\mathrm{Pt}$, than the oxygen reduction on the cathode side. The largest Pt consumer in a PEMFC is, thus, a cathode catalyst layer (CCL), which converts fluxes of protons and electrons into water with the aid of oxygen. CCL is a composite structure of interpenetrating clusters of proton-conducting ionomer (typically Nafion), voids for oxygen transport and $\mathrm{Pt} / \mathrm{C}$ particles, which transport electrons through the layer and provide the sites for the oxygen reduction reaction (ORR).

So far, the effect of $\mathrm{Pt} / \mathrm{C}$ content on the transport and kinetic properties of the CCL remains poorly understood. Experiments show that lowering of Pt content to $0.1 \mathrm{mg}_{P t} \mathrm{~cm}^{-2}$ strongly reduces the cell performance at higher current densities. Gretszler et al. showed that this lowering mathematically is equivalent to $\sim 35 \mathrm{~nm}$ bulk-like ionomer film covering the surface of $\mathrm{Pt} / \mathrm{C}$ agglomerates in the CCL. ${ }^{1}$ However, this Nafion film thickness largely exceeds the value predicted by molecular dynamics simulations $s^{2,3}$ and the recently measured value of $\simeq 7 \mathrm{~nm} .{ }^{4}$ Weber and Kusogly ${ }^{5}$ and Kusoglu et al. ${ }^{6}$ reported evidences that the structure of Nafion film covering $\mathrm{Pt} / \mathrm{C}$ particles strongly differs from the structure of bulk Nafion due to confinement effects. Recently, Freiberg et al. ${ }^{7}$ attributed increase in the cell resistance in low-Pt loaded electrodes to the diffusive oxygen transport resistance in a Nafion film covering $\mathrm{Pt} / \mathrm{C}$ agglomerates. Kulikovsky provided model-based arguments in favor of a limiting rate of oxygen adsorption on the Pt surface in low-Pt electrodes. ${ }^{8}$ This mechanism has also been considered by Kongkanand and Mathias.9

The situation with the Nafion content in the electrode is further complicated by the nonuniformities of Nafion distribution in the CCL. Orfanidi et al. ${ }^{10}$ suggested that in the standard Pt/C electrodes, the thickness of Nafion film covering Pt/C agglomerates is strongly nonuniform. They managed to homogenize this thickness by

*Electrochemical Society Member.

${ }^{\mathrm{z} E-m a i l: ~ t a t y a n a r @ h a w a i i . e d u ; ~ A . K u l i k o v s k y @ f z-j u e l i c h . d e ~}$ functionalization of carbon surface by $\mathrm{NH}_{x}$ groups. Moreover, they showed that this technique homogenizes also the Nafion distribution through the catalyst layer depth. A strong nonuniformity of proton conductivity through the CCL depth has been reported by Lefebvre, ${ }^{11}$ $\mathrm{Li}$ and Pickup ${ }^{12}$ and by Reshetenko and Kulikovsky. ${ }^{13,14}$ A detailed discussion of the effects of Nafion loading is given in a recent review of Huang et al. ${ }^{15}$

Measurements of an oxygen mass transport resistance in a low-Pt electrode have been performed using the method of limiting current density in a cell fed with diluted oxygen, ${ }^{1,16,17}$ and in a hydrogenpumped cell. ${ }^{7,9}$ Both methods enable determination of the GDL masstransfer resistance; however, measuring of the CCL oxygen transport properties by the limiting current methods is less reliable. The reason is that the limiting current is identified as a "vertical" part of the cell polarization curve. ${ }^{1,16,17}$ This part is achieved due to oxygen transport in the GDL; at this current, the oxygen concentration at the CCL/GDL interface approaches zero, and only a small CCL sub-layer located at this interface contributes to the current production. This means, that the method "feels" the oxygen transport at the CCL/GDL interface, which may differ from the transport in the bulk CCL. Moreover, this method does not allow to separate contributions of the Knudsen oxygen transport in the CCL and the microporous layer (MPL), which further reduces the accuracy of returned transport coefficients.

Below, we report characterization of low- and high-Pt loaded cathodes at various current densities based on impedance measurements, which allow us to separate the oxygen transport coefficients in the CCL and GDL. We have measured impedance spectra of PEMFCs equipped with the low- and high-Pt loaded membrane electrode assemblies (MEAs). Further, we have fitted the physics-based model for the PEMFC impedance to the measured spectra and we report the dependencies of fitting parameters on the cell current density. The model takes into account the variation of CCL proton conductivity through the electrode depth, which is quite a strong function according to our previous studies. ${ }^{13,14}$ The model also accounts for the impedance due to oxygen transport in the channel $Z_{h}$; this allowed us to fit the experimental spectra from the lowest to the highest measured frequency. A more detailed CFD-based impedance model of Bao and Bessler ${ }^{18}$ includes the impedance $Z_{h}$; however, the model below partly employs analytical solutions, and in contrast to, ${ }^{18}$ it is fast enough to perform fitting of the experimental spectra. It should be noted, that unlike the limiting current method, our impedance model works from relatively high down to low cell currents, when the whole electrode thickness contributes to current production and oxygen is transported through the entire CCL depth. 


\section{Experimental}

All electrochemical measurements were carried out using a segmented cell system and a test station developed at Hawaii Natural Energy Institute. The whole system allows us to perform simultaneous recording of spatially resolved current, voltage and impedance responses from a segmented cell which is operated as a single cell using the test station and standardized testing protocols. Such operation minimizes impacts from the segmented cell setup and represents operating conditions that are identical to real since only overall cell current or voltage is controlled by the test station. Details of the segmented cell system are presented in our previous publications. ${ }^{19,20}$

The cell was operated with commercially available $100 \mathrm{~cm}^{2}$ catalyst coated membranes (CCMs) provided by Gore. Samples with low and high platinum content were evaluated in this work. The catalyst loadings were $0.1 / 0.1$ and $0.4 / 0.4 \mathrm{mg} \mathrm{Pt} \mathrm{cm}^{-2}$ for anode/cathode and for low- and high-Pt CCM, respectively. Sigracet 25 BC (thickness $235 \mu \mathrm{m}, 80 \%$ porosity) was used as the anode and cathode gas diffusion layers (GDLs). 25 BC consists of carbon paper substrate and microporous layer with the thickness of $40-45 \mu \mathrm{m}$. Segmentation was applied for the cathode whereas the anode was unsegmented. The total active area of membrane electrode assembly (MEA) was $76 \mathrm{~cm}^{2}$. The gasket material was made of Teflon, with the thicknesses of $125 \mu \mathrm{m}$ for both electrodes and low- and high-Pt samples.

The polarization curve (VI curve) and EIS measurements were performed with $\mathrm{H}_{2} /$ air at a cell operating temperature of $80^{\circ} \mathrm{C}$. The anode/cathode conditions were $2 / 9.5$ stoichiometry, $100 / 50 \%$ relative humidity and $48.3 / 48.3 \mathrm{kPa}$ gauge backpressure. The VI curve recording was combined with spatial EIS. The selected frequency range for the EIS experiments was $0.05 \mathrm{~Hz}$ to $10 \mathrm{kHz}$ and the amplitude of the sinusoidal current signal perturbation was $2 \mathrm{~A}$, which resulted in a cell voltage response of $10 \mathrm{mV}$ or lower. Impedance spectra were measured simultaneously from 10 segments and from the whole cell.

The MEAs structure was studied by scanning electron microscopy (SEM), using Hitachi S-4800 field emission microscope with an accelerating voltage of $5 \mathrm{kV}$.

\section{Impedance Model}

The impedance model used in this work is based on the transient charge and mass conservation equations in the CCL, GDL and in the air channel. The CCL performance model has been discussed in detail in Ref. 21, while the GDL and channel model have been developed in Ref. 22. However, the latter model has been constructed assuming fast oxygen transport in the CCL. Here, we build a complete model for the CCL, GDL and channel performance which is free from this limitation. Further, the CCL model we use here takes into account the variation of proton conductivity through the electrode depth. The main model assumptions are as following.

1. The flow in the cathode channel is a plug flow (a well mixed flow with a constant velocity).

2. The oxygen transport in the catalyst and gas-diffusion layers obeys to the Fick's law with the effective diffusion coefficients $D_{o x}$ and $D_{b}$, respectively. Note that $D_{b}$ takes into account oxygen transport in the gas-diffusion media and in the microporous layer (MPL). Oxygen transport in Pt/C agglomerates is neglected (for discussion of this issue see Ref. 23).

3. The cell is operated far from equilibrium and the ORR kinetics is described by the Tafel law with the first-order dependence on the oxygen concentration.

4. The CCL proton conductivity decays exponentially with the distance from the membrane. ${ }^{13,14}$

Performance model for the CCL.-The core of the impedance model is a system of transient equations for the CCL performance: ${ }^{24-26}$

$$
C_{d l} \frac{\partial \eta}{\partial t}+\frac{\partial j}{\partial x}=-i_{*}\left(\frac{c}{c_{h}^{i n}}\right) \exp \left(\frac{\eta}{b}\right)
$$

$$
\begin{gathered}
j=-\sigma_{0} s(x) \frac{\partial \eta}{\partial x} \\
\frac{\partial c}{\partial t}-D_{o x} \frac{\partial^{2} c}{\partial x^{2}}=-\frac{i_{*}}{4 F}\left(\frac{c}{c_{h}^{i n}}\right) \exp \left(\frac{\eta}{b}\right)
\end{gathered}
$$

Here $C_{d l}$ is the double layer volumetric capacitance (per unit electrode volume, $\mathrm{F} \mathrm{cm}^{-3}$ ), $\eta$ is the positive by convention ORR overpotential, $t$ is time, $j$ is the local proton current density, $x$ is the distance from the membrane through the CCL depth, $i_{*}$ is the volumetric exchange current density $\left(\mathrm{A} \mathrm{cm}^{-3}\right), c$ is the local oxygen concentration, $c_{h}^{i n}$ is its reference concentration, $b$ is the Tafel slope, $\sigma_{0}$ is the CCL proton conductivity at the membrane interface, $s(x)$ is the conductivity shaping function, and $D_{o x}$ is the effective oxygen diffusion coefficient in the CCL.

Eq. 1 is the proton charge conservation, Eq. 2 is the Ohm's law relating the proton current density to the gradient of overpotential, and Eq. 3 is the oxygen mass conservation equation in the CCL with the Fick's law of diffusion. Note that the CCL proton conductivity is assumed to be a function of the coordinate through the CCL depth. Detailed discussion of Eqs. 1-3 is given in Ref. 21.

Substitution of Eq. 2 into Eq. 1 leads to a diffusion-type equation for the overpotential. Linearization and Fourier-transform of the resulting system yields the pair of linear equations for the smallamplitude perturbations of overpotential $\eta^{1}$ and oxygen concentration $c^{1}$ in the frequency domain (see Ref. 21 for details):

$$
\begin{gathered}
\varepsilon^{2} \frac{\partial}{\partial \tilde{x}}\left(s(\tilde{x}) \frac{\partial \tilde{\eta}^{1}}{\partial \tilde{x}}\right)=\exp \left(\tilde{\eta}^{0}\right) \tilde{c}^{1}+\left(\tilde{c}^{0} \exp \tilde{\eta}^{0}+\mathrm{i} \tilde{\omega}\right) \tilde{\eta}^{1} \\
\varepsilon^{2} \tilde{D}_{o x} \frac{\partial^{2} \tilde{c}^{1}}{\partial \tilde{x}^{2}}=\left(\exp \tilde{\eta}^{0}+\mathrm{i} \tilde{\omega} \mu^{2}\right) \tilde{c}^{1}+\tilde{c}^{0} \exp \left(\tilde{\eta}^{0}\right) \tilde{\eta}^{1}
\end{gathered}
$$

where the superscripts 0 and 1 mark the steady-state solution and the amplitude of a small harmonic perturbation, respectively,

$$
\varepsilon=\sqrt{\frac{\sigma_{0} b}{i_{*} l_{t}^{2}}}, \quad \mu=\sqrt{\frac{4 F c_{h}^{i n}}{C_{d l} b}}
$$

and the following dimensionless variables have been used

$$
\begin{aligned}
& \tilde{x}=\frac{x}{l_{t}}, \quad \tilde{t}=\frac{t}{t_{*}}, \quad \tilde{\eta}=\frac{\eta}{b}, \quad \tilde{j}=\frac{j}{j_{p}}, \quad \tilde{c}=\frac{c}{c_{h}^{i n}} \\
& \tilde{D}_{o x}=\frac{D_{o x}}{D_{*}}, \quad \tilde{Z}=\frac{Z \sigma_{0}}{l_{t}}, \quad \tilde{\omega}=\omega t_{*}
\end{aligned}
$$

Here $\omega=2 \pi f$ is the angular frequency of the applied signal, $Z$ is the impedance (see below),

$$
t_{*}=\frac{C_{d l} b}{i_{*}}, \quad j_{p}=\frac{\sigma_{0} b}{l_{t}}, \quad D_{*}=\frac{\sigma_{0} b}{4 F c_{h}^{i n}}
$$

are the scaling parameters for time, current density, and diffusion coefficient, respectively. Below, we will assume that $s(\tilde{x})$ is exponential function

$$
s=\exp (-\beta \tilde{x})
$$

where $\beta$ is the inverse characteristic scale of the exponent. Experimental and modeling study ${ }^{13,14}$ have shown that Eq. 9 provides a good fit of the spectra in the high-frequency range.

The system of Equations 4,5 subject to the boundary conditions

$$
\begin{gathered}
\tilde{\eta}^{1}(1)=\tilde{\eta}_{1}^{1},\left.\quad \frac{\partial \tilde{\eta}^{1}}{\partial \tilde{x}}\right|_{\tilde{x}=1}=0 \\
\left.\frac{\partial \tilde{c}^{1}}{\partial \tilde{x}}\right|_{\tilde{x}=0}=0, \quad \tilde{c}^{1}(1)=\tilde{c}_{b}^{1}(1)
\end{gathered}
$$

The first of Eqs. 10 fixes the perturbation amplitude $\tilde{\eta}_{1}^{1}$ of applied signal at $\tilde{x}=1$; the second one means zero proton current at the CCL/GDL interface. Eqs. 11 express zero oxygen flux through 
the membrane and continuity of the oxygen concentration at the CCL/GDL interface, respectively. Here, $\tilde{c}_{b}^{1}$ is the perturbation amplitude of the oxygen concentration in the GDL.

Oxygen transport in the GDL.-The equation for $\tilde{c}_{b}^{1}$ is obtained from the oxygen mass balance in the GDL, which in the dimensionless variables 7 has the form

$$
\mu^{2} \frac{\partial \tilde{c}_{b}}{\partial \tilde{t}}-\varepsilon^{2} \tilde{D}_{b} \frac{\partial^{2} \tilde{c}_{b}}{\partial \tilde{x}^{2}}=0
$$

where $\tilde{c}_{b}=c_{b} / c_{h}^{i n}$ and $\tilde{D}_{b}=D_{b} / D_{*}$ are the dimensionless oxygen concentration and the effective oxygen diffusion coefficient in the GDL, respectively.

Eq. 12 is linear and hence the equation for the oxygen concentration perturbation amplitude $\tilde{c}_{b}^{1}$ is

$$
\varepsilon^{2} \tilde{D}_{b} \frac{\partial^{2} \tilde{c}_{b}^{1}}{\partial \tilde{x}^{2}}=\mathrm{i} \mu^{2} \tilde{\omega} \tilde{c}_{b}^{1},
$$

The boundary conditions for this equation are

$$
\left.\tilde{D}_{b} \frac{\partial \tilde{c}_{b}^{1}}{\partial \tilde{x}}\right|_{\tilde{x}=1}=\left.\tilde{D}_{o x} \frac{\partial \tilde{c}^{1}}{\partial \tilde{x}}\right|_{\tilde{x}=1}, \quad \tilde{c}_{b}^{1}\left(1+\tilde{l}_{b}\right)=\tilde{c}_{h}^{1}
$$

where $l_{b}$ is the GDL thickness, and $\tilde{c}_{h}^{1}$ is the amplitude of the oxygen concentration perturbation in the channel. The first condition means continuity of the oxygen flux at the CCL/GDL interface, and the second one expresses continuity of the oxygen concentration perturbation at the GDL/channel interface. The equation for $\tilde{c}_{h}^{1}$ is discussed in the next section.

Oxygen transport in the channel.-The equation for $\tilde{c}_{h}^{1}$ has been derived in Ref. 22; here, for completeness, we briefly reproduce the main points of this derivation. The mass balance equation for the oxygen concentration $c_{h}$ in the channel is

$$
\frac{\partial c_{h}}{\partial t}+v \frac{\partial c_{h}}{\partial z}=\frac{1}{h} N_{b}, \quad c_{h}(t, 0)=c_{h}^{i n}
$$

where $z$ is the coordinate along the channel,

$$
N_{b}=-\left.D_{b} \frac{\partial c_{b}}{\partial x}\right|_{x=l_{t}+l_{b}}
$$

is the oxygen flux through the GDL/channel interface and $c_{h}^{i n}$ is the inlet oxygen concentration. In dimensionless form, Eq. 15 reads

$$
\xi^{2} \frac{\partial \tilde{c}_{h}}{\partial \tilde{t}}+\lambda \tilde{J} \frac{\partial \tilde{c}_{h}}{\partial \tilde{z}}=\tilde{N}_{b}, \quad \tilde{c}_{h}(\tilde{t}, 0)=1
$$

where

$$
\tilde{z}=\frac{z}{L}, \quad \xi=\sqrt{\frac{4 F h c_{h}^{i n} l_{t} i_{*}}{C_{d l} \sigma_{0} b^{2}}}
$$

$L$ is the channel length, and $J$ is the mean current density in the cell. Note that the product $\lambda \tilde{J}=\tilde{N}_{h}^{i n}$, where $\tilde{N}_{h}^{i n}$ is the dimensionless inlet oxygen flux in the channel. This flux is assumed to be fixed, i.e., in the equations below, the product $\lambda \tilde{J}$ is not affected by perturbations. Under fixed $\lambda \tilde{J}$, Eq. 16 is linear and hence an equation for the perturbation amplitude $\tilde{c}_{h}^{1}$ is

$$
\lambda \tilde{J} \frac{\partial \tilde{c}_{h}^{1}}{\partial \tilde{z}}=-\mathrm{i} \xi^{2} \tilde{\omega} \tilde{c}_{h}^{1}+\tilde{N}_{b}^{1}, \quad \tilde{c}_{h}^{1}(0)=0
$$

where $\tilde{N}_{b}^{1}$ is the perturbation amplitude of the oxygen flux at the GDL/channel interface. With the expression for $\tilde{N}_{b}^{1}$ (Ref. 22), we come to

$$
\lambda \tilde{J} \frac{\partial \tilde{c}_{h}^{1}}{\partial \tilde{z}}=A(\tilde{z}) \tilde{\eta}_{1}^{1}+\left(B(\tilde{z})-\mathrm{i} \xi^{2} \tilde{\omega}\right) \tilde{c}_{h}^{1}, \quad \tilde{c}_{h}^{1}(0)=0
$$

where $A$ and $B$ are the coefficient functions given in Appendix.
As discussed in Ref. 27, the total cell impedance is well approximated by the local impedance calculated at the point $\tilde{z}_{*}$ where the local current density equals the mean cell current density. Hence, we solve Eq. 19 , and for further calculations we take $\tilde{c}_{h}^{1}\left(\tilde{z}_{*}\right)$, where $\tilde{z}_{*}$ is a solution to equation $j_{0}=J$ :

$$
-\lambda \ln \left(1-\frac{1}{\lambda}\right) J\left(1-\frac{1}{\lambda}\right)^{\tilde{z}}=J
$$

Here, the left side is the local current density. Solving Eq. 20, we get

$$
\tilde{z}_{*}=\frac{\lambda \ln \left(f_{\lambda}\right)}{f_{\lambda}}
$$

where $f_{\lambda}=-\lambda \ln \left(1-\frac{1}{\lambda}\right)$.

Reduction of the performance equations and impedance.-Eq. 19 contains a single unknown function, $\tilde{c}_{h}^{1}$. At large cell current, this equation can only be solved numerically. The rest of the problem reduces to numerical solution of the system 4, 5. Indeed, Eq. 13 and the system 4,5 are linked through the boundary conditions at the CCL/GDL interface, Eqs. 11,14. Solving Eq. 13 and setting $\tilde{x}=1$ in the solution, we get

$$
\begin{aligned}
\tilde{c}_{b}^{1}(1)= & -\left.\frac{\tanh \left(\mu \tilde{l}_{b} \sqrt{\mathrm{i} \tilde{\omega} /\left(\varepsilon^{2} \tilde{D}_{b}\right)}\right)}{\mu \sqrt{\mathrm{i} \tilde{\omega} \tilde{D}_{b} / \varepsilon^{2}}} \tilde{D}_{o x} \frac{\partial \tilde{c}^{1}}{\partial \tilde{x}}\right|_{\tilde{x}=1} \\
& +\frac{\tilde{c}_{h}^{1}}{\cosh \left(\mu \tilde{l}_{b} \sqrt{\mathrm{i} \tilde{\omega} /\left(\varepsilon^{2} \tilde{D}_{b}\right)}\right)}
\end{aligned}
$$

where $\tilde{c}_{h}^{1}=\tilde{c}_{h}^{1}\left(\tilde{z}_{*}\right)$, as discussed in the previous section. With this, the second of Eqs. 11 transforms into the Robin-type boundary condition:

$$
\begin{gathered}
\tilde{c}^{1}(1)+\left.\frac{\tanh \left(\mu \tilde{l}_{b} \sqrt{\mathrm{i} \tilde{\omega} /\left(\varepsilon^{2} \tilde{D}_{b}\right)}\right)}{\mu \sqrt{\mathrm{i} \tilde{\omega} \tilde{D}_{b} / \varepsilon^{2}}} \tilde{D}_{o x} \frac{\partial \tilde{c}^{1}}{\partial \tilde{x}}\right|_{\tilde{x}=1} \\
=\frac{\tilde{c}_{h}^{1}}{\cosh \left(\mu \tilde{l}_{b} \sqrt{\mathrm{i} \tilde{\omega} /\left(\varepsilon^{2} \tilde{D}_{b}\right)}\right)}
\end{gathered}
$$

Thus, with $\tilde{c}_{h}^{1}$ at hand, solution to the system 4,5 with the boundary conditions discussed above yields the local shapes $\tilde{c}^{1}(\tilde{x})$ and $\tilde{\eta}^{1}(\tilde{x})$.

The static shapes $\tilde{\eta}^{0}$ and $\tilde{c}^{0}$ appearing in Eqs. 4,5 are solutions to the dimensionless steady-state version of the system 1-3:

$$
\begin{aligned}
\varepsilon^{2} \frac{\partial}{\partial \tilde{x}}\left(s \frac{\partial \tilde{\eta}^{0}}{\partial \tilde{x}}\right) & =\tilde{c}^{0} \exp \tilde{\eta}^{0}, \quad \tilde{\eta}^{0}(0)=\tilde{\eta}_{0}^{0},\left.\quad \frac{\partial \tilde{\eta}^{0}}{\partial \tilde{x}}\right|_{\tilde{x}=1}=0 \\
\varepsilon^{2} \tilde{D}_{o x} \frac{\partial^{2} \tilde{c}^{0}}{\partial \tilde{x}^{2}} & =\tilde{c}^{0} \exp \tilde{\eta}^{0},\left.\quad \frac{\partial \tilde{c}^{0}}{\partial \tilde{x}}\right|_{\tilde{x}=0}=0, \quad \tilde{c}^{0}(1)=1-\frac{\tilde{j}_{0}}{\tilde{j}_{l i m}}
\end{aligned}
$$

where $j_{\text {lim }}=4 F D_{b} c_{h}^{i n} / l_{b}$.

Solution to the system 24,25 requires setting of the static overpotential $\tilde{\eta}_{0}^{0}$. However, the impedance measurements are usually performed at the fixed static current density $\tilde{j}_{0}$. The system 24,25 can be reformulated in terms of the proton current density, ${ }^{28}$ which yields

$$
\frac{\partial^{2} \tilde{j}^{0}}{\partial \tilde{x}^{2}}-\left(\frac{\left(\tilde{j}_{0}-\tilde{j}^{0}\right)}{\tilde{D}_{o x} \tilde{c}}-\frac{\tilde{j}}{s}\right) \frac{\partial \tilde{j}^{0}}{\partial \tilde{x}}=0, \quad \tilde{j}^{0}(0)=\tilde{j}_{0}, \quad \tilde{j}^{0}(1)=0
$$

$$
\tilde{D}_{o x} \frac{\partial \tilde{c}^{0}}{\partial \tilde{x}}=\tilde{j}_{0}-\tilde{j}^{0}, \quad \tilde{c}^{0}(1)=1-\frac{\tilde{j}_{0}}{\tilde{j}_{l i m}}
$$

The system 26,27 is equivalent to the system 24,25 ; however, under fixed $\tilde{j}_{0}$ numerical solution of equations 26,27 does not require iterations. With the shapes of $\tilde{j}^{0}(\tilde{x})$ and $\tilde{c}^{0}(\tilde{x})$ at hand, the overpotential $\tilde{\eta}^{0}$ 
is calculated according to

$$
\tilde{\eta}^{0}(\tilde{x})=\ln \left(-\frac{\varepsilon^{2}}{\tilde{c}^{0}} \frac{\partial \tilde{j}^{0}}{\partial \tilde{x}}\right)
$$

which follows from the static version of Eq. 1 .

Numerical solution of the system 4,5 with the boundary conditions 10,23 , and the first of Eqs. 11 yields the amplitude of ovepotential perturbation $\tilde{\eta}^{1}(\tilde{x})$. Note that the oxygen transport in the GDL and channel is included into the system 4, 5 through the boundary condition 23. Finally, the impedance of the cathode side ("CCL+GDL+channel") is calculated according to

$$
\tilde{Z}=-\left.\frac{\tilde{\eta}^{1}}{\partial \tilde{\eta}^{1} / \partial \tilde{x}}\right|_{\tilde{x}=0, \tilde{z}=\tilde{z}_{*}}
$$

Numerical details.-Fitting has been performed in Maple environment using the matrix form of a built-in Maple procedure NonlinearFit. This routine takes as an input a procedure which returns the local sum of squares of the form

$$
\left(Z_{r e, i}^{\text {model }}-Z_{r e, i}^{e x p}\right)^{2}+\left(Z_{i m, i}^{\text {model }}-Z_{i m, i}^{\text {exp }}\right)^{2}, \quad i=1 . . N
$$

where $Z_{r e}, Z_{i m}$ stand for the real and imaginary parts of the impedance, the superscripts model and exp denote the model and experimental values, respectively, and $N$ is the total number of points in the experimental spectrum. Note that the model impedance in Eq. 30 is calculated for the same frequency, as the experimental impedance. Model components of impedance have been calculated from Eq. 29, where $\tilde{\eta}^{1}$ has been determined from numerical solution of the system 4,5. The static shapes $\tilde{\eta}^{0}$ and $\tilde{c}^{0}$ have been calculated from numerical solution of the system 26,27 and Equation 28. The systems of complex ODE's have been converted to equivalent real systems for the real and imaginary components of the unknown functions; the resulting systems have been solved using a built-in Maple procedure dsolve. The static shapes $\tilde{\eta}^{0}$ and $\tilde{c}^{0}$ have been updated after every $10-$ th iteration with NonlinearFit. Total number of iterations has typically been around 100; for a single spectrum it takes less than an hour on a 1.7-GHz notebook.

\section{Results and Discussion}

SEM pictures of the high-and low-Pt MEAs are shown in Figure 1. As can be seen, the two MEAs differ by the thickness of the catalyst layer: in agreement with the ratio of catalyst loadings in the two cells, in the low-Pt cell, the CCL thickness is roughly four times less, than in the high-Pt cell (Figure 1). The geometrical and operating parameters for the two cells are collected in Table I.

As $\lambda$ in our experiments has been sufficiently large, only spectra of the whole cell have been processed. In general, processing of the local spectra improves statistics of the results; however, local oxygen concentration at the individual segments is unknown, which lowers the accuracy of the local spectra fitting.

Six parameters have been declared as the fitting ones: the Tafel slope $b$, the proton conductivity at the CCL/membrane interface $\sigma_{0}$, the double layer capacitance $C_{d l}$, the oxygen diffusion coefficients in the CCL $D_{o x}$, and in the GDL $D_{b}$, and the oxygen stoichiometry $\lambda$. Though $\lambda$ is known from the experiment, we claimed it as a fitting parameter as variable $\lambda$ effectively takes into account under-rib oxygen transport in the meander flow field. For both the cells, parameter $\beta$ in Eq. 9 have been fixed at 6.5; this value has been determined by fitting the spectra for the current density of $50 \mathrm{~mA} \mathrm{~cm}^{-2}$ with $\beta$ as a fitting parameter. Control fitting of the spectrum for $j_{0}=400 \mathrm{~mA} \mathrm{~cm}^{-2}$ with $\beta$ declared as a fitting parameter gave $\beta=6.41$ and only marginal variation of the other parameters.

Figures 2, 3 show the experimental and fitted model Nyquist spectra of a low- and high-Pt loaded cells, respectively. Figures 4, 5 show the Bode plots corresponding to the current densities of 50 and $400 \mathrm{~mA} \mathrm{~cm}^{-2}$ for the low-Pt and high-Pt cells, respectively. As can be seen, the quality of fitting is good. Note that the fitted model points
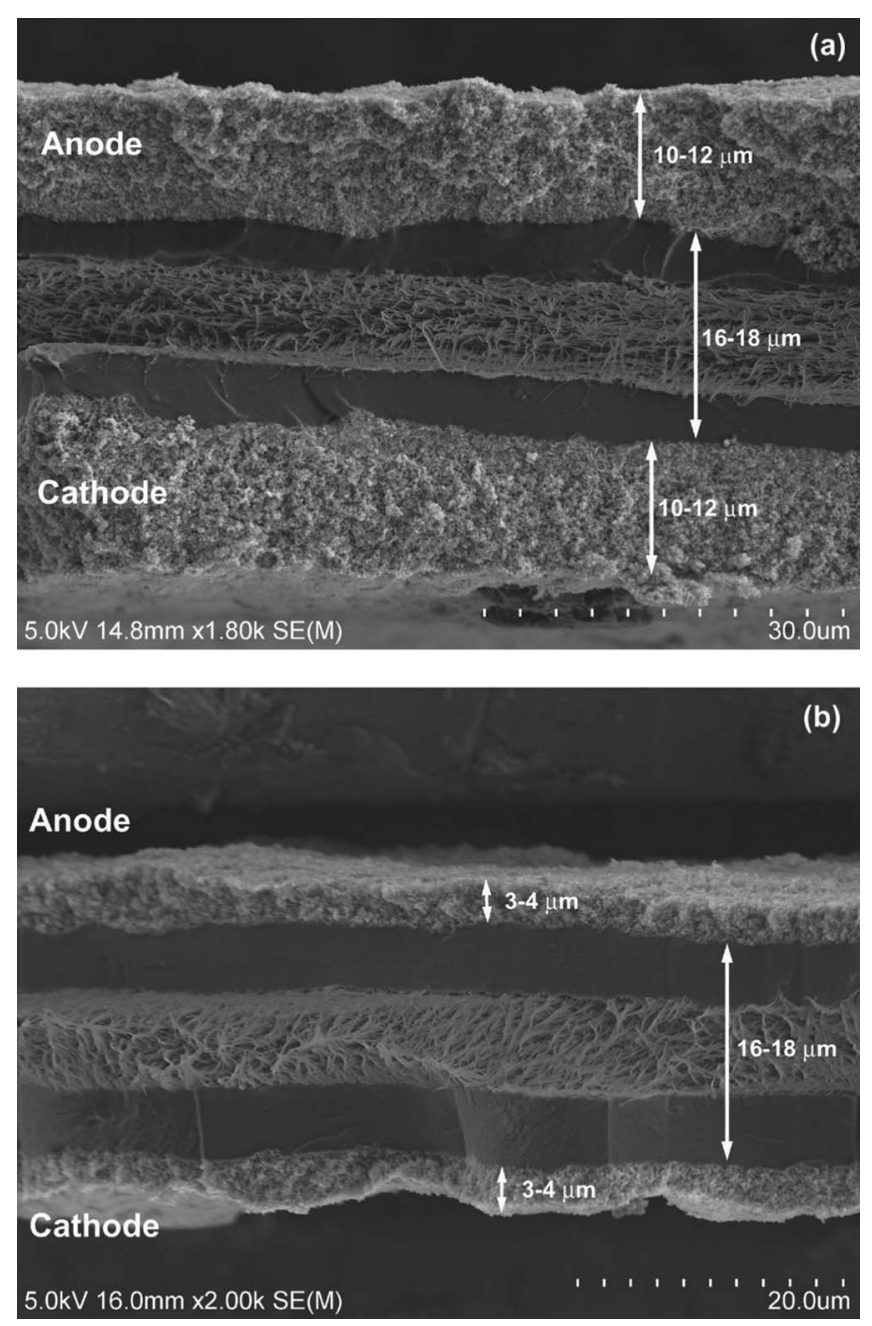

Figure 1. Scanning electron microscopy pictures of (a) the high-Pt and (b) the low-Pt MEAs.

(open circles) in Figures 2-5 are shown for the same frequencies, as the experimental points (filled circles).

The dependence of fitting parameters on the cell current density $j_{0}$ is shown in Figures 6-8. In the low- and high-Pt cells, the Tafel slope exhibits a weak linear growth with $j_{0}$; in the low-Pt cell, the Tafel slope is up to $30 \%$ higher, than in the high-Pt cell (Figure 6a). In both the cells, mean proton conductivity rapidly increases with the cell current, and in the low-Pt cell this parameter is lower, than in the high-Pt cell (Figure 6b). The double layer capacitance is nearly the same in both the cells, and it decreases with $j_{0}$ reaching a plateau at $\simeq 10 \mathrm{~F} \mathrm{~cm}^{-3}$ (Figure $6 \mathrm{c}$ ). In both the cells, the CCL oxygen diffusivity

Table I. Geometrical and operating parameters for the two cells. $\mathrm{A} / \mathrm{C}$ stands for anode/cathode.

\begin{tabular}{lcc} 
& $\begin{array}{c}\text { Low-Pt } \\
\text { cell }\end{array}$ & $\begin{array}{c}\text { High-Pt } \\
\text { cell }\end{array}$ \\
\hline Catalyst loading A/C, $\operatorname{mg}_{P t} \mathrm{~cm}^{-2}$ & $0.1 / 0.1$ & $0.4 / 0.4$ \\
Catalyst layer thickness $l_{t}, \mu \mathrm{m}$ & 2.7 & 12 \\
Gas diffusion layer thickness $l_{b}, \mu \mathrm{m}$ & 230 & 235 \\
Exchange current density $i_{*}, \mathrm{~A} \mathrm{~cm}^{-3}$ & \multicolumn{2}{c}{$-/ 10^{-3}$} \\
(assumed) & \multicolumn{2}{c}{$2 / 9.5$} \\
Flow stoichiometry A/C & \multicolumn{2}{c}{$100 \% / 50 \%$} \\
Relative humidity A/C & \multicolumn{2}{c}{$1.48 / 1.48$}
\end{tabular}



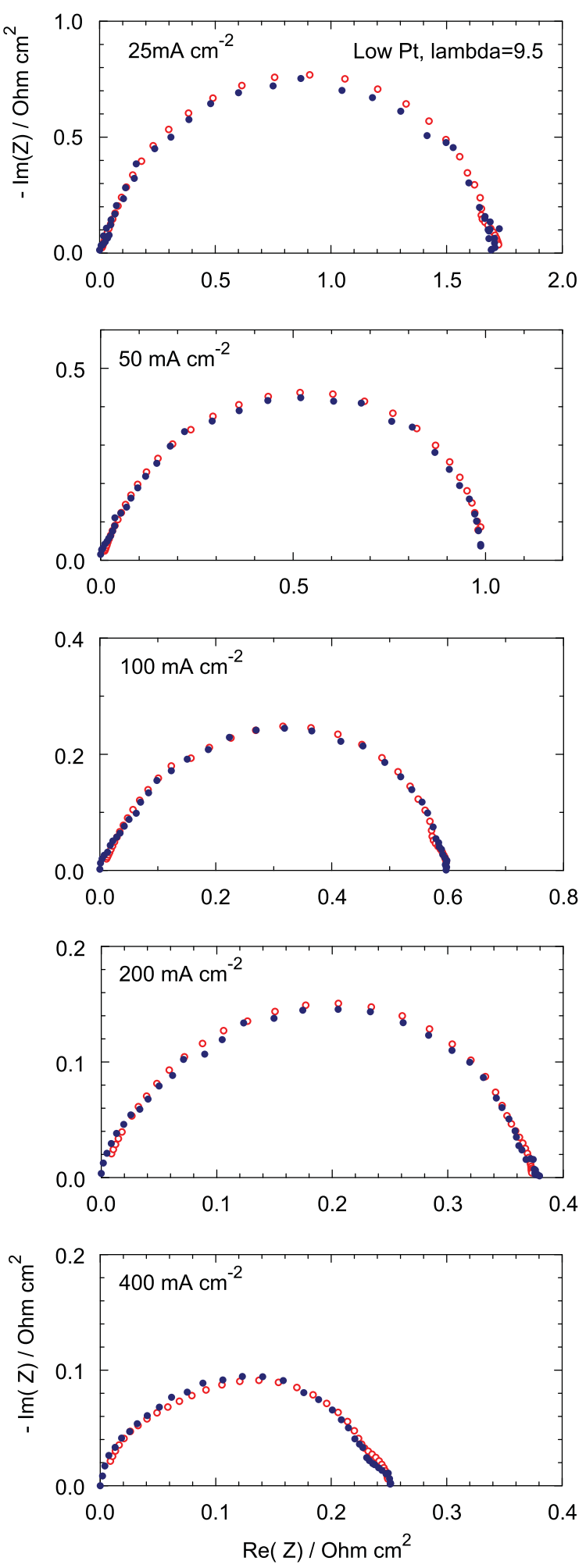

Figure 2. Experimental (filled points) and fitted model (open circles) spectra of the low-Pt fuel cell for the indicated current densities.

$D_{o x}$ exhibits linear growth with $j_{0}$. In the low-Pt cell, $D_{o x}$ is nearly an order of magnitude lower, than in the high-Pt cell (Figure 7a, see below). The GDL oxygen diffusivity exhibits a weak growth with $j_{0}$ in a high-Pt cell, and it is nearly independent on $j_{0}$ in a low-Pt cell (Figure 8a).

Note that the model above takes into account doubling of the effective Tafel slope at higher currents due to finite rates of proton
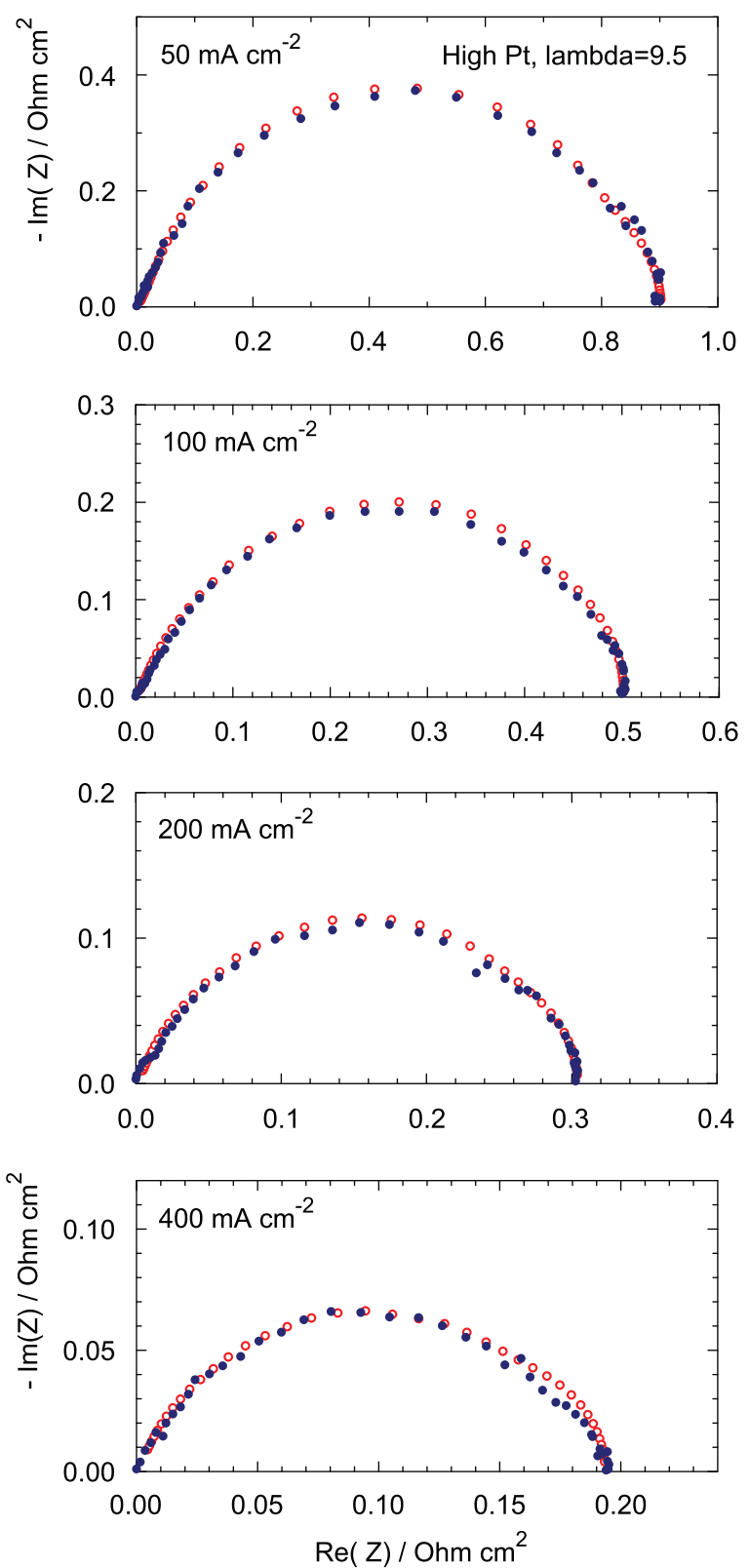

Figure 3. Experimental (filled points) and fitted model (open circles) spectra of the high-Pt fuel cell for the indicated current densities.

and/or oxygen transport in the cell. In other words, Figure 6a shows a true "kinetic" value of this parameter. The growth of the Tafel slope with the cell current density could be explained by change of the ORR preferential location on a Pt surface. Pt nanoparticle is a multifaceted object with the facets having different crystallographic structure. ${ }^{29}$ With the growth of the cell current density, the ORR preferential location may shift from one facet type to another, which translates into variation of the Tafel slope. Another reason for a growth of $b$ with $j_{0}$ could be "cleaning" of Pt surface from oxides with the lowering of the cell potential. ${ }^{30}$ Note that the higher Tafel slope in the low-Pt cell is in line with the observation that this parameter increases with $j_{0}$ (Figure 6a). Indeed, at a fixed current density per geometrical cell area $j_{0}$, the current density per unit Pt active surface (ECSA) in the low-Pt cell would be higher, than in the high-Pt cell.

The growth of the mean proton conductivity of the CCL with $j_{0}$ has been reported in Ref. 31; possible explanation is the growth of a number of current-conducting channels in the Nafion film covering the $\mathrm{Pt} / \mathrm{C}$ agglomerates. ${ }^{31}$ Lower proton conductivity of a low-Pt CCL 

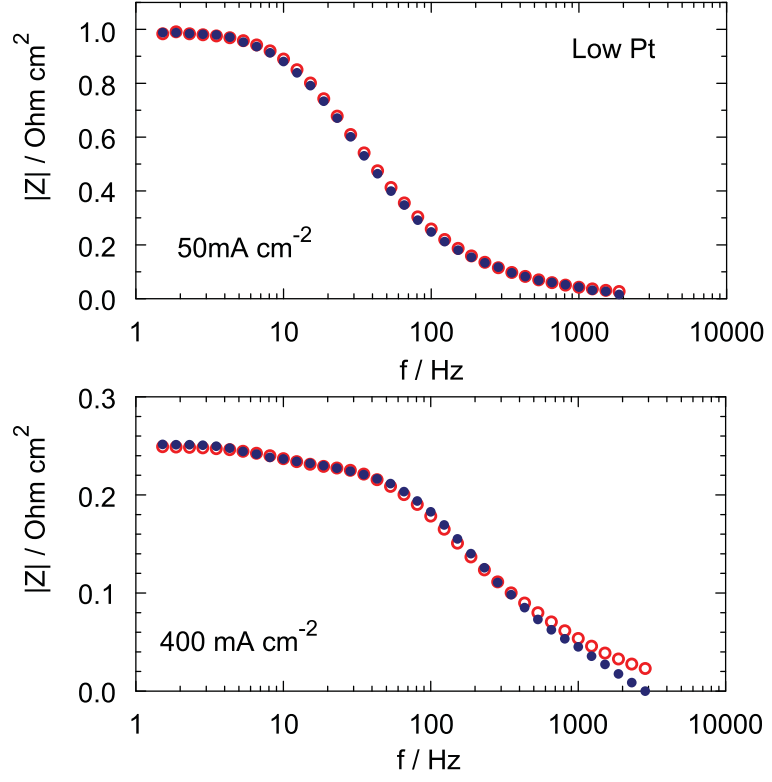

Figure 4. Bode plots of the experimental (filled points) and fitted model (open circles) spectra of the low-Pt fuel cell for the indicated current densities.

as compared to the conductivity of a high-Pt CCL can be explained as following. The conductivity is determined mainly by the HF shape of impedance spectra; it shows the rate of the proton transport to the catalyst sites. The proton pathway includes the Nafion and/or water film covering the $\mathrm{Pt} / \mathrm{C}$ agglomerates and, possibly, the proton transport inside the agglomerates. Molecular dynamics simulations of Malek et al. $^{2,3}$ show that larger amount of Pt increases the amount of water on the surface of $\mathrm{Pt} / \mathrm{C}$ particles, which may facilitate the proton transport to the Pt surface. Further, according to ${ }^{3} \mathrm{Pt}$ content strongly affects the structure and connectivity of Nafion cluster in the CCL.

In contrast to the proton conductivity, the volumetric double layer capacitance of both CCLs is nearly the same (Figure 6c). Indeed, the volumetric density of $\mathrm{Pt}$ particles in both the electrodes is the same, which translates into close values of $C_{d l}$. Lowering of $C_{d l}$ with the growth of the cell current is most probably due to increasing
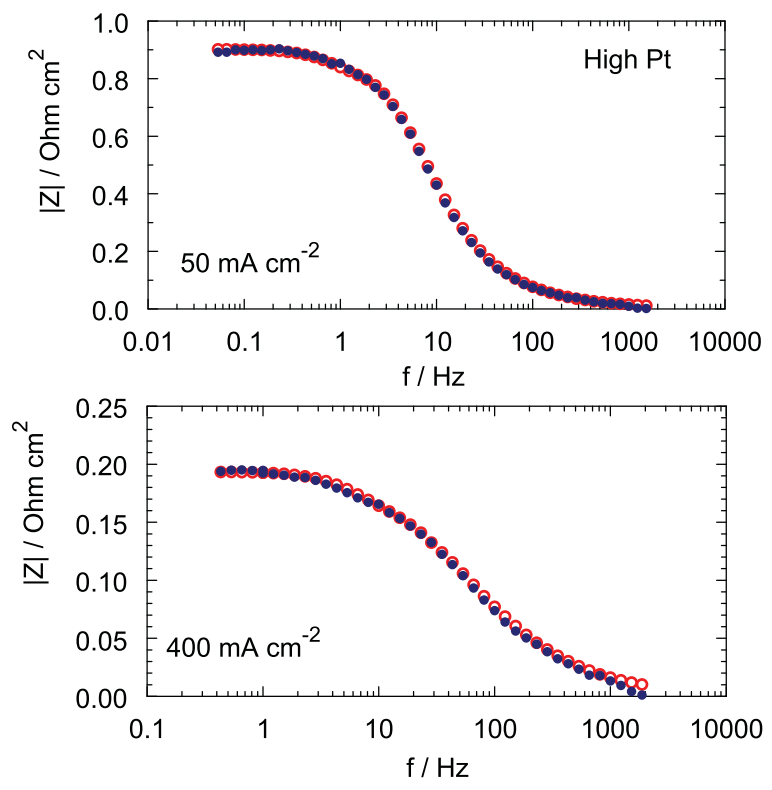

Figure 5. Bode plots of the experimental (filled points) and fitted model (open circles) spectra of the high-Pt fuel cell for the indicated current densities.
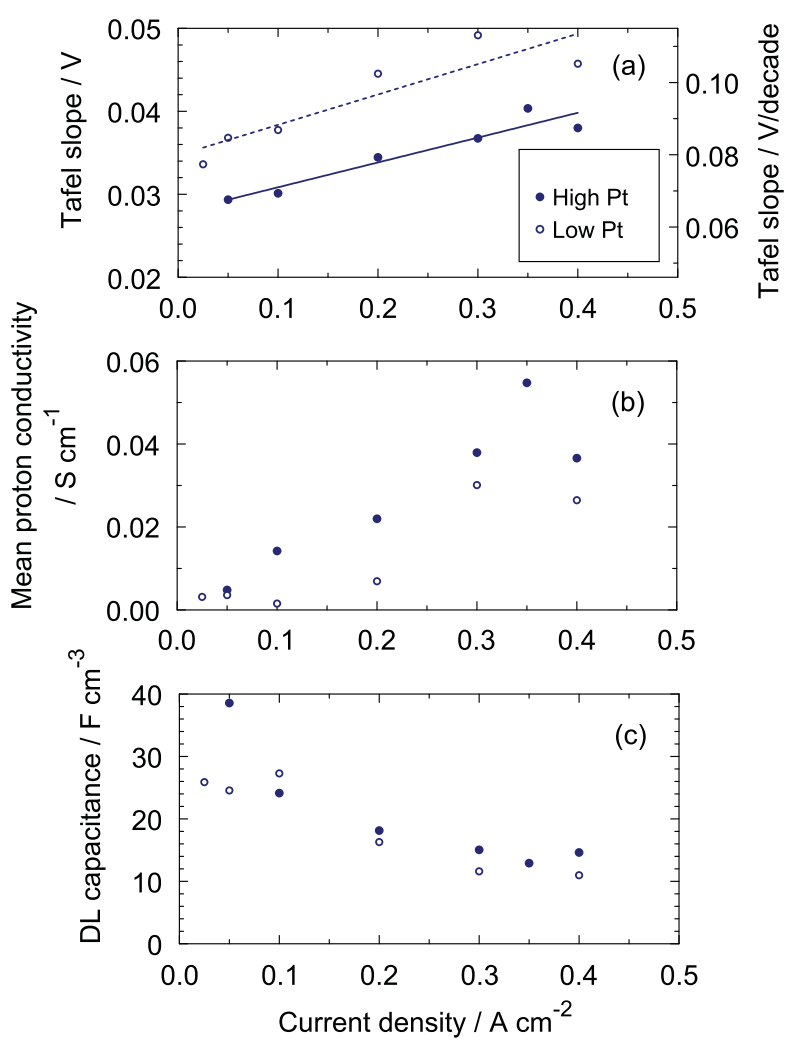

Figure 6. Fitting parameters for the low-Pt (open circles) and high-Pt (filled circles) loaded cells. (a) The ORR Tafel slope, (b) the double layer capacitance, (c) the mean through the CCL depth proton conductivity.

with $j_{0}$ amount of liquid water in the catalyst layer. Part of the $\mathrm{Pt}$ electroactive surface appears to be flooded, which reduces the double layer capacitance of the electrode.

The CCL oxygen diffusivity increases with the cell current, and in the low-Pt cell this parameter is nearly an order of magnitude lower, than in the high-Pt cell (Figure 7a). However, due to the 4fold difference in the electrode thickness (Table I), the mass transfer
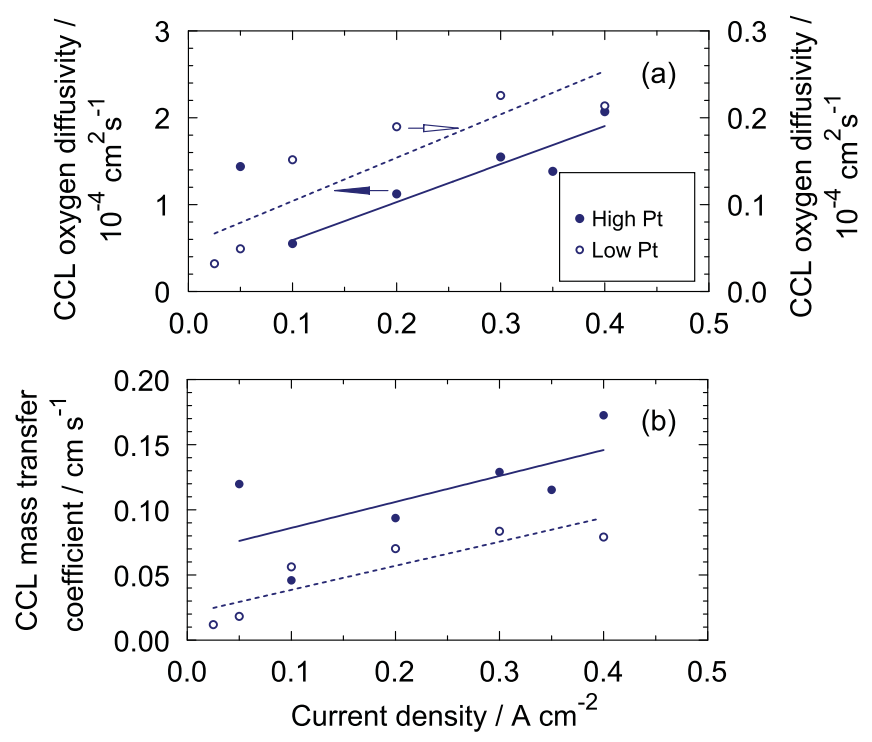

Figure 7. Fitting parameters for the low-Pt (open circles) and high-Pt (filled circles) loaded cells. (a) Effective oxygen diffusion coefficient in the CCL, (b) Mass transfer coefficient in the CCL. 

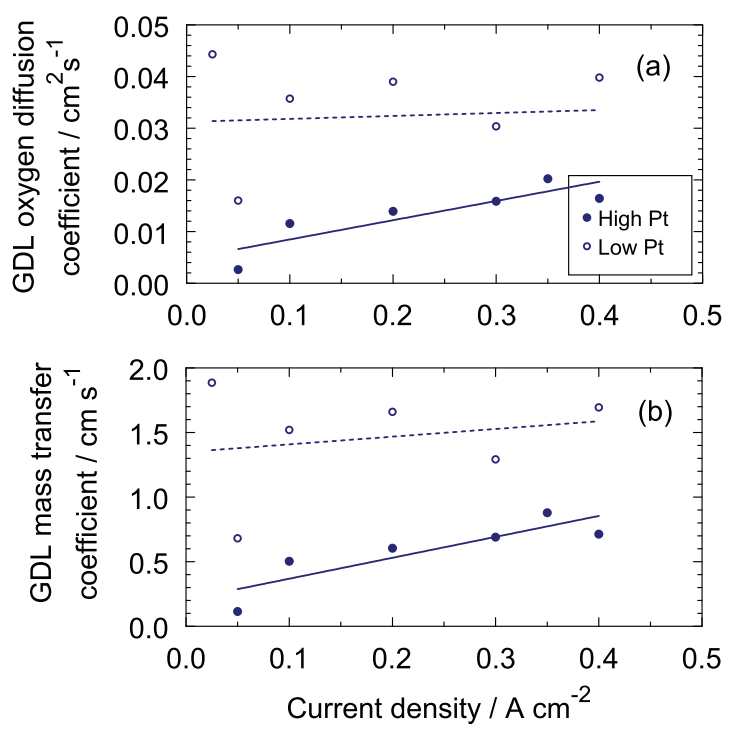

Figure 8. Fitting parameters for the low-Pt (open circles) and high-Pt (filled circles) loaded cells. (a) Effective oxygen diffusion coefficient in the GDL, (b) Mass transfer coefficient in the GDL.

coefficients in both the electrodes are close to each other (Figure 7b). The oxygen mass transfer coefficient $k_{o x, i}$ is calculated according to

$$
k_{o x, i}=\frac{D_{i}}{l_{i}},
$$

where $i=\{\mathrm{CCL}, \mathrm{GDL}\}$, and $l_{i}$ and $D_{i}$ are the thickness and the oxygen diffusivity of the $i$-th layer. Note that in our model, $D_{o x}$ is an effective parameter; Eq. 3 only implies the Fick's law for the oxygen flux in the CCL, not specifying the physical nature of $\mathrm{O}_{2}$ transport. In particular, the model does not separate the oxygen transport in the void pores and in the Nafion film covering Pt/C agglomerates. Attempt to apply the mode ${ }^{23}$ to separate the oxygen diffusion through the Nafion film has failed. With the expected Nafion film thickness on the order of $10 \mathrm{~nm}$ and the oxygen diffusion coefficient in Nafion on the order of $10^{-6} \mathrm{~cm}^{2} \mathrm{~s}^{-1}$ (Ref. 32), the characteristic angular frequency for oxygen transport in the Nafion film is about $10^{5} \mathrm{~Hz}$. This frequency is far above the frequency range used in experiments $(50 \mathrm{mHz}-10 \mathrm{kHz})$, and the impedance model ${ }^{23}$ cannot capture this processes.

As discussed by Malek et al., ${ }^{3} \mathrm{Pt}$ affects the structure of Nafion and void clusters in the CCL shifting the pore size distribution to larger pores, which facilitates the transport of gaseous oxygen through the electrode. Another reason for lower $D_{o x}$ in a low-Pt cell could be limiting rate of the oxygen adsorption on the Pt surface. In a low-Pt electrode, this process may increase the slope of a high-current part of the cell polarization curve, like a diffusive transport limitation. ${ }^{8}$ Much lower $D_{o x}$ in the low-Pt electrode (Figure 7a) can, therefore, be attributed either to the influence of Pt loading on the structure of Nafion cluster, ${ }^{3}$ or to the limiting rate of oxygen adsorption on the $\mathrm{Pt}$ surface. The physical origin of the linear growth of $D_{o x}$ with $j_{0}$ is not clear and it requires further studies. Note that this growth correlates with the linear increase of the Tafel slope in the CCL (Figure 6a). If the growth of the Tafel slope is caused by cleaning of Pt surface from oxides, than the growth of $D_{o x}$ could be caused by increasing amount of sites available for oxygen adsorption, which translates into higher effective oxygen diffusivity of the CCL.

Different values of the GDL oxygen diffusivity $D_{b}$ in the lowand high-Pt cells (Figure 8a) seemingly is due to variations in the preparation conditions of the two MEAs. As the thicknesses of the GDLs used in low- and high-Pt cells are nearly the same, the respective mass transfer coefficients are close to each other (Figure 8a). Comparing Figures 7 and 8 we see that in both the cells, the mass transfer coefficient of the CCL is an order of magnitude lower, than this coefficient of the GDL. The GDL mass transfer coefficient of a high-Pt cell (Figure 8b) agrees with the limiting current method measurements reported by Reshetenko and St-Pierre. ${ }^{17}$

\section{Conclusions}

Impedance spectra of the low-Pt $\left(0.1 / 0.1 \mathrm{mg}_{P t} \mathrm{~cm}^{-2}\right)$ and high-Pt $\left(0.4 / 0.4 \mathrm{mg}_{P t} \mathrm{~cm}^{-2}\right)$ cells have been measured for the cell current density $j_{0}$ in the range of 50 to $400 \mathrm{~mA} \mathrm{~cm}^{-2}$. The impedance model, which takes into account nonuniformity of the proton conductivity through the CCL depth and the oxygen transport in the channel has been fitted to the spectra from the lowest to the highest frequencies. The $j_{0}$-dependencies of the ORR Tafel slope, mean proton conductivity, the double layer capacitance, and of the CCL and the GDL oxygen diffusivities are presented.

- The ORR Tafel slope in both the CCLs linearly increases with the cell current density; in the low-Pt cell, this parameter is about $20 \%$ higher, than in the high-Pt cell.

- The mean through the CCL depth proton conductivity increases with $j_{0}$ by an order of magnitude, from the value of less than $0.005 \Omega^{-1} \mathrm{~cm}^{-1}$ at $j_{0}=50 \mathrm{~mA} \mathrm{~cm} \mathrm{~cm}^{-2}$ up to $0.04 \Omega^{-1} \mathrm{~cm}^{-1}$ at $j_{0}=400 \mathrm{~mA} \mathrm{~cm}^{-2}$. In a low-Pt electrode this parameter is lower, than in the high-Pt electrode.

- In both the cells, the double layer capacitance per unit electrode volume is nearly the same, decreasing from about $30 \mathrm{~F} \mathrm{~cm}^{-3}$ at small currents to the plateau on the level of $10 \mathrm{~F} \mathrm{~cm}^{-3}$ at large currents.

- The effective oxygen diffusion coefficient in the CCL of the low-Pt electrode is nearly an order of magnitude lower, than this coefficient in a high-Pt electrode. However, due to four times lower low-Pt CCL thickness, the oxygen mass transfer coefficients in both the catalyst layers are of the same order of magnitude.

- The GDL oxygen diffusion coefficient in the low-Pt electrode is three to two times higher, than in the high-Pt electrode.

- The concerted linear growth of the Tafel slope and the CCL effective oxygen diffusivity $D_{o x}$ with the cell current density could be attributed to "cleaning" of Pt surface from oxides with the decreasing of the cell potential. Pt cleaning means more sites available for oxygen adsorption, which translates into higher $D_{o x}$.

\section{Acknowledgments}

T. Reshetenko is grateful to funding from US Office of Naval Research (N00014-12-1-0496), US Army Research Office (W911NF15-1-0188) and the Hawaiian Electric Company for ongoing support of the Hawaii Sustainable Energy Research Facility. The authors also thank Gunter Randolf for valuable support with the system operation.

\section{Appendix: Coefficient Functions in Eq. 19}

The coefficient functions $A$ and $B$ in Eq. 19 are given by ${ }^{22}$

$$
\begin{aligned}
A(\tilde{z})= & \frac{\mu \phi^{3} \sin (\phi) \psi \tilde{c}_{1}^{0}}{\cos \left(\mu \tilde{l}_{b} \psi\right)\left(\tilde{c}_{1}^{0} \mu \phi^{2} \psi+\tilde{j}_{0} \tan \left(\mu \tilde{l}_{b} \psi\right) \phi \sin (\phi)\right)} \\
B(\tilde{z})= & -\frac{\mu \phi \sin (\phi) \psi \tilde{j}_{0}}{\cos ^{2}\left(\mu \tilde{l}_{b} \psi\right)\left(\tilde{c}_{1}^{0} \mu \phi^{2} \psi+\tilde{j}_{0} \tan \left(\mu \tilde{l}_{b} \psi\right) \phi \sin (\phi)\right)} \\
& +\mu \sqrt{-\mathrm{i} \tilde{\omega} \tilde{D}_{b} / \varepsilon^{2}} \tan \left(\mu \tilde{l}_{b} \psi\right)
\end{aligned}
$$

where

$$
\begin{gathered}
\phi=\sqrt{-\tilde{j}_{0}-\mathrm{i} \tilde{\omega} / \varepsilon^{2}}, \quad \psi=\sqrt{-\mathrm{i} \tilde{\omega} /\left(\varepsilon^{2} \tilde{D}_{b}\right)} \\
\tilde{c}_{1}^{0}=\left(1-\frac{1}{\lambda}\right)^{\tilde{z}}-\frac{\tilde{j}_{0} \tilde{l}_{b}}{\tilde{D}_{b}}
\end{gathered}
$$

\section{List of Symbols}

Marks dimensionless variables

$b \quad$ Tafel slope, $\mathrm{V}$ 
$C_{d l} \quad$ Double layer volumetric capacitance, $\mathrm{F} \mathrm{cm}^{-3}$

$c \quad$ Oxygen molar concentration in the CCL, $\mathrm{mol} \mathrm{cm}^{-3}$

$c_{b} \quad$ Oxygen molar concentration in the GDL, $\mathrm{mol} \mathrm{cm} \mathrm{cm}^{-3}$

$c_{h} \quad$ Oxygen molar concentration in the channel, $\mathrm{mol} \mathrm{cm} \mathrm{cm}^{-3}$

$D_{o x} \quad$ Effective oxygen diffusion coefficient in the CCL, $\mathrm{cm}^{2} \mathrm{~s}^{-1}$

$D_{b} \quad$ Effective oxygen diffusion coefficient in the GDL, $\mathrm{cm}^{2} \mathrm{~s}^{-1}$

$F \quad$ Faraday constant, $\mathrm{C} \mathrm{mol}^{-1}$

$f \quad$ Regular frequency, $\mathrm{Hz}$

$J \quad$ Mean current density in the cell, $\mathrm{A} \mathrm{cm}^{-2}$

$j \quad$ Local proton current density in the CCL, $\mathrm{A} \mathrm{cm}^{-2}$

$j_{0} \quad$ Local cell current density, $\mathrm{A} \mathrm{cm}^{-2}$

$j_{p} \quad$ Characterisitc cell current density for proton transport in the

CCL, Eq. 8, $\mathrm{A} \mathrm{cm}^{-2}$

Channel depth, $\mathrm{cm}$

Imaginary unit

ORR Volumetric exchange current density, $\mathrm{A} \mathrm{cm}^{-3}$

Channel length, $\mathrm{cm}$

Gas-diffusion layer thickness, $\mathrm{cm}$

Catalyst layer thickness, $\mathrm{cm}$

Exponential shaping function for the proton conductivity in the CCL, Eq. 9

Time, $\mathrm{s}$

Characteristic time of double layer charging, s, Eq. 8

flow velocity in the channel, $\mathrm{cm} \mathrm{s}^{-1}$

Coordinate through the cell, $\mathrm{cm}$

Total impedance of the cathode side, $\Omega \mathrm{cm}^{2}$

\section{Greek}

$\beta \quad$ Dimensionless parameter, Eq. 9

$\varepsilon \quad$ Newman's dimensionless reaction penetration depth, Eq. 6

$\eta \quad$ Local ORR overpotential (positive by convention), $\mathrm{V}$

$\mu \quad$ Dimensionless parameter, Eq. 6

$\sigma_{0} \quad$ CCL ionic conductivity at the membrane interface, $\Omega^{-1} \mathrm{~cm}^{-1}$

Angular frequency $(\omega=2 \pi f), \mathrm{s}^{-1}$

\section{Subscripts}

$0 \quad$ Membrane/CCL interface

$1 \quad$ CCL/GDL interface

$b \quad$ GDL

$t \quad$ Catalyst layer

* Characteristic value

\section{Superscripts}

$0 \quad$ Steady-state value

1 Small-amplitude perturbation

\section{ORCID}

Andrei Kulikovsky (D) https://orcid.org/0000-0003-1319-576X

\section{References}

1. T. A. Greszler, D. Caulk, and P. Sinha, The impact of platinum loading on oxygen transport resistance, J. Electrochem. Soc., 159, F831 (2012).

2. K. Malek, M. Eikerling, Q. Wang, T. Navessin, and Zh. Liu, Self-organization in catalyst layers of polymer electrolyte fuel cells, J. Phys. Chem, 111, 13627 (2007).
3. K. Malek, T. Mashio, and M. Eikerling, Microstructure of catalyst layers in PEM fuel cells redefined: A computational approach, Electrocatal., 2, 141 (2011).

4. M. Lopez-Haro, L. Guetaz, T. Printemps, A. Morin, S. Escribano, P.-H. Jouneau, P. Bayle-Guillemaud, F. Chandezon, and G. Gebel, Three-dimensional analysis of Nafion layers in fuel cell electrodes, Nature Comm., 5, (2014).

5. A. Z. Weber and A. Kusoglu, Unexplained transport resistances for low-loaded fuelcell catalyst layers, J. Mater. Chem. A, 2, 17207 (2014).

6. A. Kusoglu, D. Kushner, D. K. Paul, K. Karan, M. A. Hickner, and A. Z. Weber, Impact of substrate and processing on confinement of Nafion thin films, Adv. Funct. Mater. 24, 4763 (2014).

7. A. T. S. Freiberg, M. C. Tucker, and A. Z. Weber, Polarization loss correction derived from hydrogen local-resistance measurement in low Pt-loaded polymer-electrolyte fuel cells, Electrochem. Comm., 79, 14 (2017).

8. A. A. Kulikovsky, Polarization curve of a PEM fuel cell with the account of a finite rate of oxygen adsorption on Pt surface, Int. J. Hydrogen Energy, 39, 19018 (2014).

9. A. Kongkanand and M. F. Mathias, The priority and challenge of high-power performance of lowplatinum proton-exchange membrane fuel cells, Phys. Chem. Lett., 7, 1127 (2016).

10. A. Orfanidi, P. Madkikar, A. El-Sayed, G. S. Harzer, T. Kratky, and H. A. Gasteiger, The key to high performance low Pt loaded electrodes, J. Electrochem. Soc., 164, F418 (2017).

11. M. C. Lefebvre, R. B. Martin, and P. G. Pickup, Characterization of ionic conductivity profiles within proton exchange membrane fuel cell gas diffusion electrodes by impedance spectroscopy, Electrochem. Solid State Lett., 2, 259 (1999).

12. G. Li and P. G. Pickup, Ionic conductivity of pemfc electrodes. effect of Nafion loading, J. Electrochem. Soc., 150, C745 (2003).

13. T. Reshetenko and A. Kulikovsky, Impedance spectroscopy study of the PEM fuel cell cathode with nonuniform nafion loading, J. Electrochem. Soc., 164, E3016 (2017).

14. A. A. Kulikovsky, Impedance of a PEM fuel cell cathode with nonuniform ionomer loading: Analytical and numerical study, J. Electroanal. Chem., 789, 174 (2017).

15. J. Huang, Z. Li, and J. Zhang, Review of characterization and modeling of polymer electrolyte fuel cell catalyst layer: The blessing and curse of ionomer, Front. Energy, 11, 334 (2017).

16. D. R. Baker, D. A. Caulk, K. C. Neyerlin, and M. W. Murphy, Measurement of oxygen transport resistance in PEM fuel cells by limiting current methods, J. Electrochem. Soc., 156, B991 (2009).

17. T. V. Reshetenko and J. St-Pierre, Separation method for oxygen mass transport coefficient in gas and ionomer phases in PEMFC GDE, J. Electrochem. Soc., 161, F1089 (2014).

18. C. Bao and W. G. Bessler, Two-dimensional modeling of a polymer electrolyte membrane fuel cell with long flow channel. Part II. physics-based electrochemical impedance analysis, J. Power Sources, 278, 675 (2015).

19. T. Reshetenko and A. Kulikovsky, PEM fuel cell characterization by means of the physical model for impedance spectra, J. Electrochem. Soc., 162, F627 (2015).

20. T. Reshetenko and A. Kulikovsky, Comparison of two physical models for fitting PEM fuel cell impedance spectra measured at a low air flow stoichiometry, J. Electrochem. Soc., 163, F238 (2016).

21. A. A. Kulikovsky, A physical model for the catalyst layer impedance, J. Electroanal. Chem., 669, 28 (2012).

22. A. Kulikovsky and O. Shamardina, A model for PEM fuel cell impedance: Oxygen flow in the channel triggers spatial and frequency oscillations of the local impedance, J. Electrochem. Soc., 162, F1068 (2015)

23. A. Kulikovsky, Can we quantify oxygen transport in the Nafion film covering an agglomerate of Pt/C particles?, J. Electrochem. Soc., 164(4), F379 (2017).

24. J. S. Newman and C. W. Tobias, Theoretical analysis of current distribution in porous electrodes, J. Electrochem. Soc., 109, 1183 (1962).

25. M. Eikerling and A. A. Kornyshev, Electrochemical impedance of the cathode catalyst layer in polymer electrolyte fuel cells, J. Electroanal. Chem., 475, 107 (1999).

26. A. A. Kulikovsky, The regimes of catalyst layer operation in a fuel cell, Electrochimica Acta, 55, 6391 (2010)

27. A. Kulikovsky, A fast low-current model for impedance of a PEM fuel cell cathode at low air stoichiometry, J. Electrochem. Soc., 164(9), F911 (2017)

28. A. Kulikovsky, A model for impedance of a PEM fuel cell cathode with poor electron conductivity, J. Electroanal. Chem, 801, 122 (2017).

29. M. Shao, A. Peles, and K. Shoemaker, Electrocatalysis on platinum nanoparticles: Particle size effect on oxygen reduction reaction activity, Nano Lett., 11, 3714 (2011).

30. Y. Liu, M. Mathias, and J. Zhang, Measurement of platinum oxide coverage in a proton exchange membrane fuel cell, Electrochem. Solid State Lett., 13, B1 (2010).

31. T. Reshetenko and A. Kulikovsky, Variation of PEM fuel cell physical parameters with current: Impedance spectroscopy study, J. Electrochem. Soc., 163(9), F1100 (2016).

32. V. A. Sethuraman, S. Khan, J. S. Jur, A. T. Haug, and J. Weidner, Measuring oxygen, carbon monoxide and hydrogen sulfide diffusion coefficient and solubility in Nafion membranes, Electrochimica Acta, 54, 6850 (2009). 